\title{
The influence of spacing between two submerged bodies on wave loads of SWATH
}

\author{
Guoqing Feng $^{\mathrm{a}}$, Xueyun Jiang ${ }^{\mathrm{b}}$, Huilong Ren $^{\mathrm{c}}$, Qingyue Zhang ${ }^{\mathrm{d}}$ \\ College of ShipBuliding Engineering, Harbin Engineering University, Harbin, 150001, China \\ a771955943@qq.com
}

\begin{abstract}
Keywords: SWATH; 3-D wave loads; long-term forecast; short-term forecast; the spacing between two submerged bodies.
\end{abstract}

\begin{abstract}
The determination of the spacing between two submerged bodies is of great significance in preliminary design stage of SWATH. And this paper focus on researching the influence of spacing between two submerged bodies on SWATH wave loads. Wave loads forecast are made for three different loading conditions, which are normal loading, full loading and maximum loading. For each loading condition, no parameter except the spacing of two submerged bodies is changed from the available data of a certain SWATH in practical project to obtain a series of SWATH computation models. By means of 3-D frequency domain, wave load forecast of different computation models is made to obtain the transfer function, long-term and short-term forecast value, then the comparisons between the value of wave load forecast and that of ABS and CCS specifications are done. By comparing the wave load forecast value of different calculation models, the degree and characteristics of impact which the spacing between two submerged bodies has on the wave loads are obtained, thus providing a reference for the preliminary design of SWATH.
\end{abstract}

\section{Introduction}

With the rapid development of SWATH in the field of shipbuilding, the study of SWATH wave loads has gradually developed and deepened in recent years. Currently, researches about SWATH wave loads are mainly focused on the following: the estimation and empirical formulas of design loads, the dangerous working conditions, the hydrodynamic pressure and motion response characteristics. In preliminary design stage of SWATH ship, the determination of spacing between two submerged bodies is very important, which has seldom been studied in the shipbuilding field. Actually, the underwater part of SWATH mainly consists of two submerged bodies and two pillar bodies, which can provide buoyancy for the whole vessel. Based on source-sink distribution theory, two submerged bodies can be regards as two symmetrically arranged ellipsoids. And the presence of one submerged body will act as an additional wall for the other, while solving the velocity potential. Thus, different spacing between two submerged bodies will surely lead to different wave loads.

Therefore, this article focus on researching the influence of spacing between two submerged bodies on SWATH wave loads under three loading conditions. For each loading condition, spacing is the only variable, thus a series computation models with different spacing are obtained. Then COMPASS-WALCS-BASIC software is adopted to do wave loads forecast for those computing models. By comparing the wave load forecast results of different computation models, the influence of spacing between two submerged bodies on SWATH wave loads can be obtained, which can provide a reference for the preliminary design of SWATH ship.

\section{Computation parameters}

To compute the sectional wave loads, 21 transverse sections and a longitudinal section are selected. And all the transverse sections distribute along the captain uniformly, while the longitudinal section locates in longitudinal section of the ship.

In regular waves computing, the computed speed is $6 \mathrm{kn}$, and the computed wave interval amounts to $15^{\circ}$, while 36 frequencies were used ( $0.2 \mathrm{rad} / \mathrm{s}$ with steps of $0.05 \mathrm{rad} / \mathrm{s}$ up to $\left.2.0 \mathrm{rad} / \mathrm{s}\right)$. Due to space 
limitations, only transfer functions of relevant wave loads of partial frequencies under full loading condition are illustrated in Figs .1 4.

Given the results of regular wave calculations, wave loads in irregular waves can be obtained based on relevant mathematical statistics principles. And the wave parameters of short-term forecast are shown in table 1, while NO.34 North Atlantic waves scatter plots recommended by IACS are selected in the long-term forecasts. In the long-term forecasts, the exceedance probability is $10^{-8}$ and the point spread function is $\frac{2}{\pi} \cos ^{2} \theta$.

Table 1. Wave parameters of short-term forecast

\begin{tabular}{ccc}
\hline Scale of state of sea & $\mathrm{H}_{\mathrm{S}}(\mathrm{m})$ & $\mathrm{T}_{\mathrm{P}}(\mathrm{s})$ \\
\hline 4 & 2.5 & 6.8 \\
5 & 4 & 7.5 \\
6 & 6 & 9.6 \\
7 & 9 & 11.6 \\
\hline
\end{tabular}

\section{Presentation of the results}

Due to space limitations, only transfer functions of relevant wave loads of partial frequencies under full loading condition are illustrated in Figs.3 6. And when the vessel is in full loading condition, the short-term wave loads forecast of different calculation models under different scale of state of sea are illustrated in Figs.5 8.

Then, comparison between the long-term forecast values of WALCS and specification values of CCS and ABS is shown in Table 2.

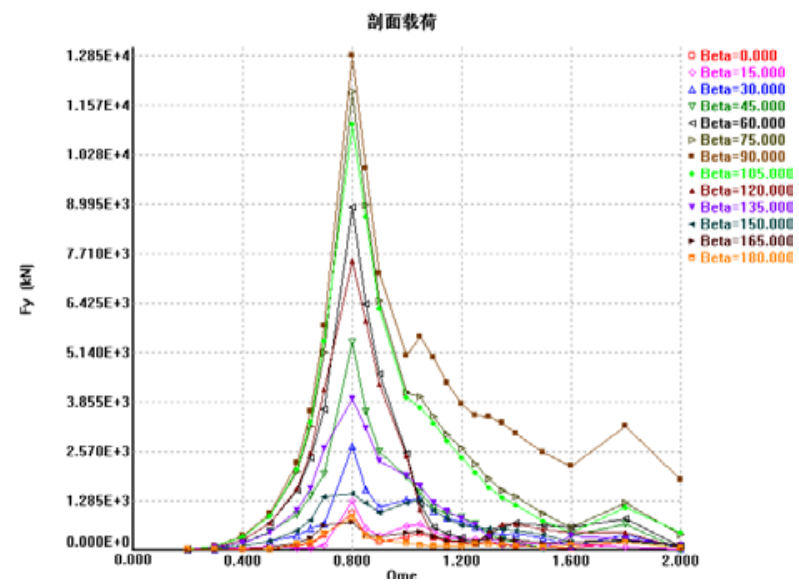

Fig.1 Transfer function of horizontal split force

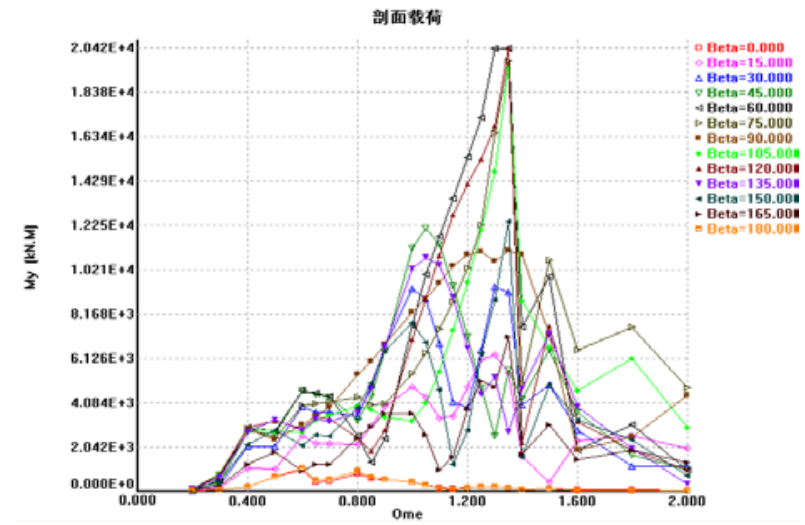

Fig.3 Transfer function of sync pitch torsional moment

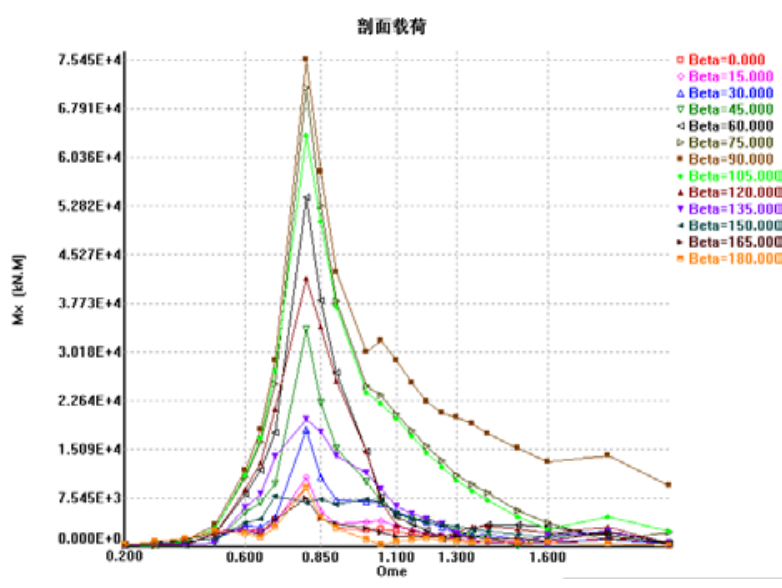

Fig.2 Transfer function of transverse bending moment

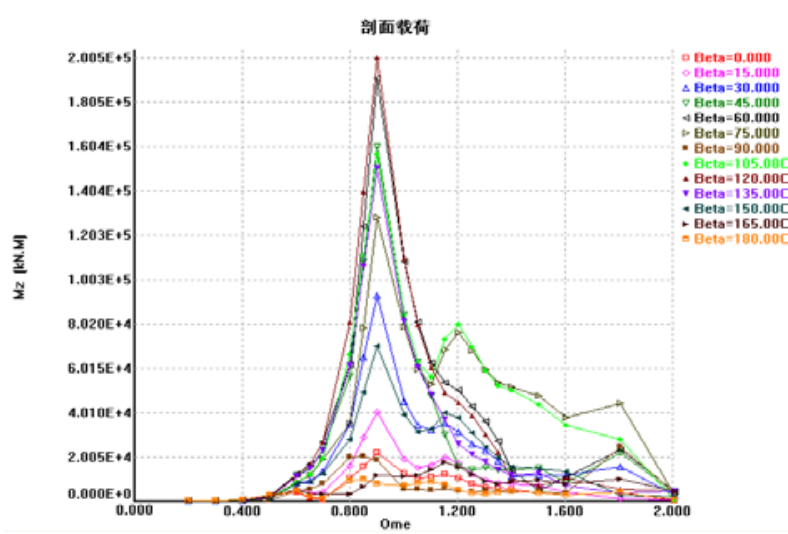

Fig.4 Ttransfer function of horizontal torsional moment 
Table 2. Long-term forecast values and specification values of CCS and ABS

\begin{tabular}{cccc}
\hline Wave loads & Compare form & Wave loads value & Ratio \\
\hline \multirow{2}{*}{ horizontal split force } & WALCS/CCS & $4.7 \times 10^{4} / 5.2 \times 10^{4}$ & $89.0 \%$ \\
& WALCS /ABS & $4.7 \times 10^{4} / 5.4 \times 10^{4}$ & $87.0 \%$ \\
transverse bending & WALCS /CCS & $4.7 \times 10^{5} / \times 10^{5}$ & $89.0 \%$ \\
moment & WALCS /ABS & $4.7 \times 10^{5} / 5.4 \times 10^{5}$ & $87.0 \%$ \\
sync pitch torsional & WALCS /CCS & $5.5 \times 10^{4} / 5.4 \times 10^{4}$ & $101.8 \%$ \\
moment & WALCS /ABS & - & - \\
horizontal torsional & WALCS /CCS & $5.6 \times 10^{5} / 4.5 \times 10^{5}$ & $124.0 \%$ \\
moment & WALCS /ABS & $5.6 \times 10^{5} / 5.9 \times 10^{5}$ & $94.0 \%$ \\
\hline
\end{tabular}

According to Table 2, the wave loads forecast valus of WALCS are very close to the computed results of CCS 《Guidelines of Small Water plane Area Twin Hull Craft》 (2005) and ABS 《Guide for Building and Classing SWATH vessels》 (1999) , thus verifying the correctness of the computation result by WALCS.

Figs. 9 12 show the short-term wave loads forecast of each computation model under three different loading conditions.

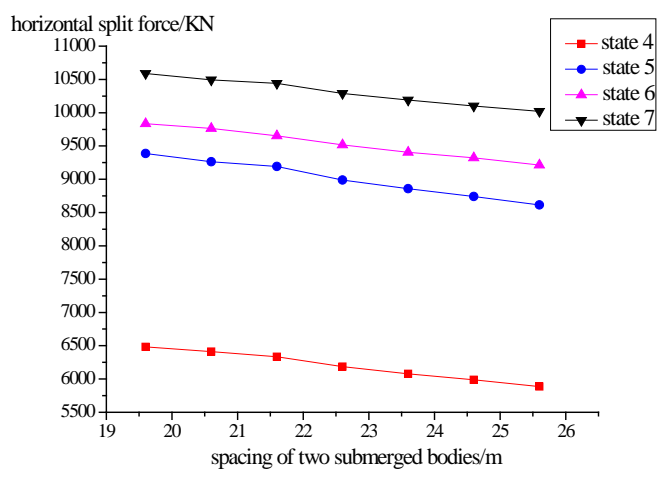

Fig.5 Short-term value of horizontal split force

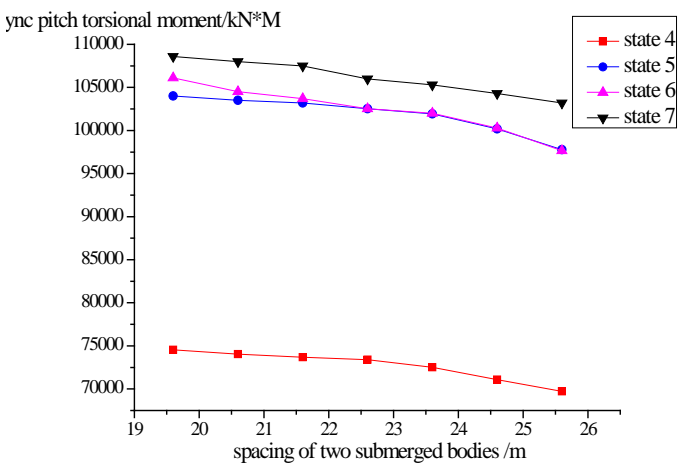

Fig.7 Short-term value of sync pitch torsional moment

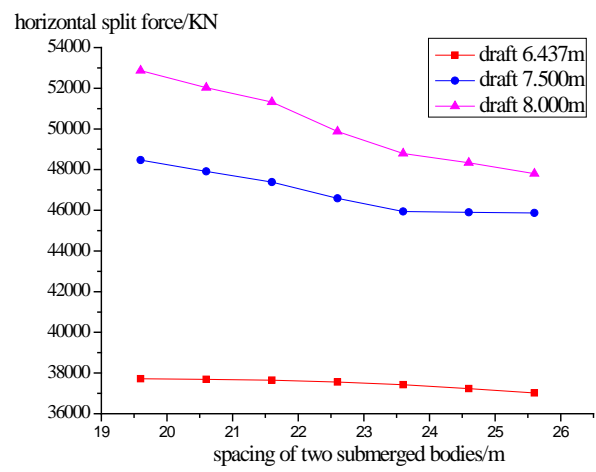

Fig.9 long-term value of horizontal split force Fig.10 long-term value of transverse bending

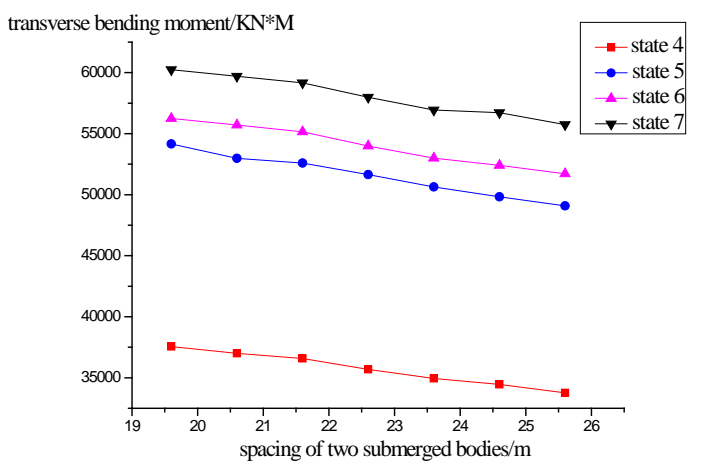

Fig.6 Short-term value of transverse bending moment

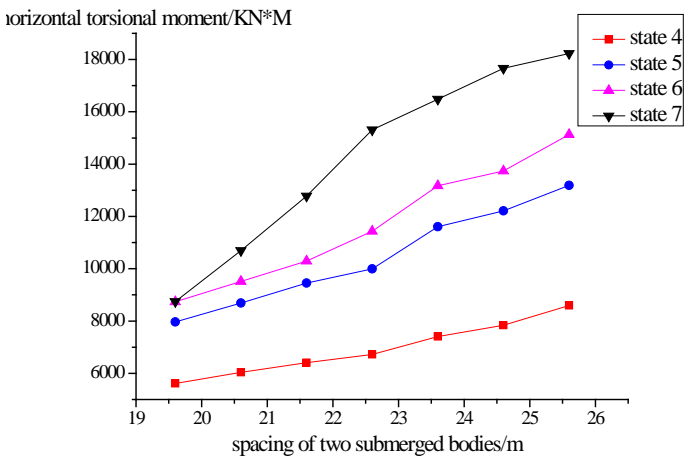

Fig.8 Short-term value of horizontal torsional moment

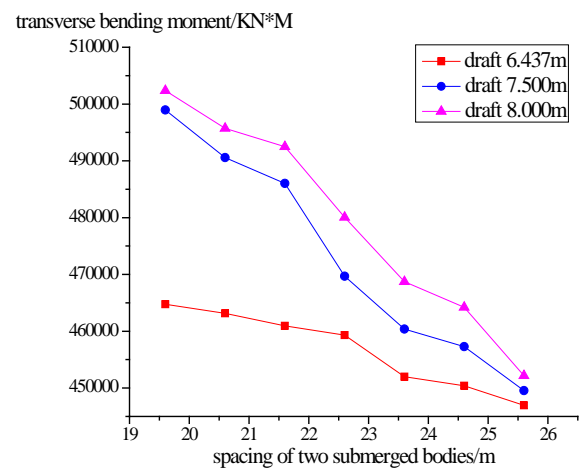




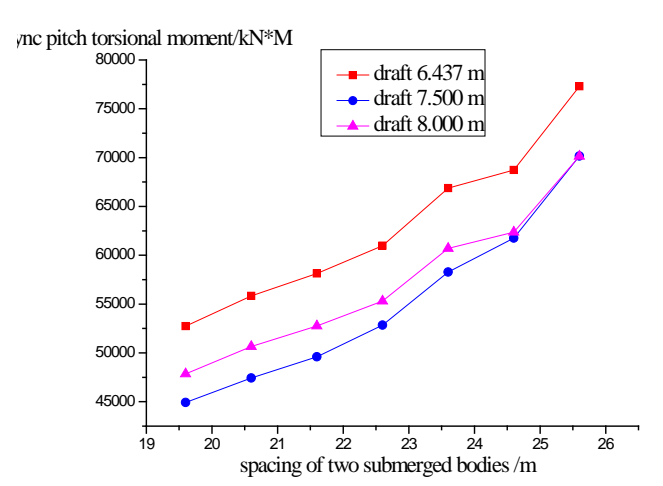

Fig.11 long-term value of sync pitch torsional moment

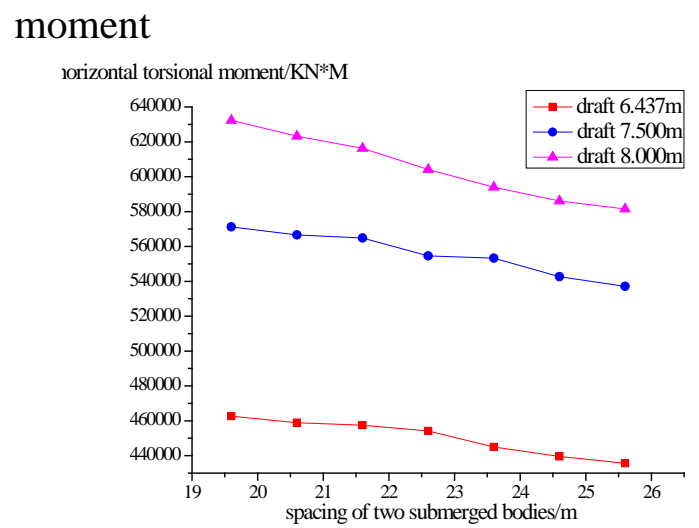

Fig.12 long-term value of horizontal torsional moment

\section{Conclusions}

Based on the short-term and long-term wave loads forecast of different computation models, the following conclusions can be drawn:

(1) From the results of short-term and long-term wave loads forecast, it can be clearly concluded that the spacing between two submerged bodies has small effect on the horizontal split force, transverse bending moment, horizontal torsional moment and longitudinal bending moment, which verifies the rationality that CCS and ABS didn't take the spacing as an impact factor in the wave load estimation formula. Though, those wave loads share a common variation: they would increase when the spacing between two submerged bodies decrease, which is very useful for the preliminary design of SWATH. (2)Different from above wave loads mentioned in(1), the spacing has relatively large impact on the sync pitch torsional moment, and the bigger the spacing is, the bigger the moment will be.

(3)When comparing the short-term and long-term wave loads forecast of the same model under different loading conditions, it can be additionally concluded that the bigger the draft is, the bigger all the wave loads will be. However, the specific influence that the draft has on wave loads may need further research.

\section{Acknowledgements}

This paper is funded by 973 funded projects, No. 2011CB013703, China. This paper would not be possible to accomplish without the fruitful advice from Professor Guo-qing Feng.

\section{References}

[1] J.R. Lin, L.G. Shi, G.H. You and J.Y. Qian. The Method for Evaluating the Design Wave Loads, J. Shipbuilding of China. 1(2008)1-5.

[2] H. Ren, Loading response of wave motion of SWATH ship, J. Journal of Ship Mechanics.1(2003)24-51.

[3] Y. Chen, Y.L. Yue, J.R Lin, W.P. Yu. Research on the Design Loads of the SWATH, J. Journal of Ship Mechanics. 16(2012)101-107.

[4] F. Wu, Z.J. Chen, J.B. Liu, Y.S. Liu. Hydrodynamic calculations in waves of SWATH, J. Ship Science and Technology. 26(2004)17-21.

[5] Y.C. Geng, J.J. Hu, X.K. Gu, X.L. Wang, E.R. Qi, The investigation of wave loads for fast trimaran, J. Ship Science and Technology. 12(2013) 5-11.

[6] CCS, Guidelines of Small Water plane Area Twin Hull Craft, 2005.

[7] ABS, Guide for Building and Classing SWATH vessels, 1999. 